\title{
Culprit or Bystander: Defective Mitophagy in Alzheimer's Disease
}

\author{
Chenglong Xie 1,2, Yahyah Aman², Bryan A. Adriaanse ${ }^{3}$, M. Zameel Cader ${ }^{3}$, \\ Hélène Plun-Favreau ${ }^{4}$, Jian Xiao ${ }^{5}$ and Evandro F. Fang ${ }^{2,6 *}$
}

${ }^{1}$ Department of Neurology, The First Affiliated Hospital of Wenzhou Medical University, Wenzhou, China, ${ }^{2}$ Department of Clinical Molecular Biology, University of Oslo, Akershus University Hospital, Lørenskog, Norway, ${ }^{3}$ Weatherall Institute of Molecular Medicine, University of Oxford, Oxford, United Kingdom, ${ }^{4}$ Queen Square Multiple Sclerosis Centre, Department of Molecular Neuroscience, UCL Queen Square Institute of Neurology, University College London, London, United Kingdom, ${ }^{5}$ Molecular Pharmacology Research Center, School of Pharmaceutical Sciences, Wenzhou Medical University, Wenzhou, China, ${ }^{6}$ The Norwegian Centre on Healthy Ageing (NO-Age), Oslo, Norway

Mitophagy is a selective engulfment and degradation of damaged mitochondria through the cellular autophagy machinery, a major mechanism responsible for mitochondrial quality control. Increased accumulation of damaged mitochondria in the Alzheimer's disease (AD) human brain are evident, although underlying mechanisms largely elusive.

\section{OPEN ACCESS}

Edited by:

Jochen H. M. Prehn,

Royal College of Surgeons in Ireland,

Ireland

Reviewed by:

Amalia M. Dolga,

University of Groningen, Netherlands

Jianbo Sun,

Sun Yat-sen University, China

${ }^{*}$ Correspondence: Evandro F. Fang e.f.fang@medisin.uio.no; evandrofeifang@yahoo.com

Specialty section:

This article was submitted to

Cell Death and Survival,

a section of the journal

Frontiers in Cell and Developmental

Biology

Received: 06 August 2019

Accepted: 23 December 2019

Published: 17 January 2020

Citation:

Xie C, Aman Y, Adriaanse BA,

Cader MZ, Plun-Favreau H, Xiao J and Fang EF (2020) Culprit or

Bystander: Defective Mitophagy

in Alzheimer's Disease.

Front. Cell Dev. Biol. 7:391.

doi: 10.3389/fcell.2019.00391
Recent studies indicate impaired mitophagy may contribute to the accumulation of damaged mitochondria in cross-species AD animal models and in AD patient PSSderived neurons. Studies from AD highlight feed-forward vicious cycles between defective mitophagy, and the principal AD pathological hallmarks, including amyloid- $\beta$ plaques, tau tangles, and inflammation. The concomitant and intertwined connections among those hallmarks of $A D$ and the absence of a real humanized $A D$ rodent model present a challenge on how to determine if defective mitophagy is an early event preceding and causal of Tau/A $\beta$ proteinopathies. Whilst further studies are required to understand these relationships, targeting defective mitophagy holds promise as a new therapeutic strategy for AD.

Keywords: Alzheimer's disease, mitophagy, aging, neuroprotection, memory

\section{MOLECULAR MECHANISMS OF MITOPHAGY AND ITS ROLES IN NEUROPLASTICITY}

Mitophagy is a highly conserved cellular process of removing damaged or superfluous mitochondria to maintain mitochondrial homeostasis (Pickrell and Youle, 2015; Scheibye-Knudsen et al., 2015; Fang et al., 2016b; McWilliams et al., 2016; Fivenson et al., 2017). In neurons, accumulation of damaged mitochondria is noxious to cellular function and survival. Mitophagy, at physiological level, maintains neuroplasticity and the functions of glial cells (Gustafsson and Dorn, 2019). Recent findings in human cell lines and multiple animal models have extended our knowledge in the molecular mechanisms of mitophagy from the PINK1-Parkin pathway, to the PINK1-independent pathways, including pathways that depend on NIP3-like protein X (NIX), B-cell lymphoma 2 interacting protein 3 (BNIP3), B-cell lymphoma 2-like 13 (BCL2L13), FK506 binding protein 8 (FKBP8), prohibitin (PHB2), breast cancer gene 1 protein (NBR1), optineurin (OPTN), calcium binding and coiled-coil domain 2 (NDP52), Autophagy and Beclin 1 Regulator 1 (AMBRA1), Tax1 binding protein 1 (TAX1BP1), FUN14 domain-containing protein 1 (FUNDC1), 
PGAM family member 5 (PGAM5), Nipsnap Homolog 1 (NIPSNAP1), NIPSNAP2, among others (Fivenson et al., 2017; Kerr et al., 2017; Palikaras et al., 2018; Lou et al., 2019; Princely Abudu et al., 2019).

The PINK-1-dependent mitophagy is one of the wellcharacterized mitophagy pathways, with mutations of PINK1 associated to familial Parkinson's disease (PD) (Plun-Favreau and Hardy, 2008; Gandhi et al., 2009; Burchell et al., 2013; Pickrell and Youle, 2015). Under physiological conditions, mitochondrial membrane potential (MMP) drives mitochondrial import of the $63 \mathrm{kDa}$ full length PINK1. Presenilin-associated rhomboid-like protein (PARL) is an inner mitochondrial membrane (IMM) protease. PARL cuts the mitochondrial targeting sequence (MTS) and trans-membrane domain of PINK1, leading to the cytosolic release of the $\mathrm{N}$-terminal-deleted PINK1 ( $\Delta$ N-PINK1) (Deas et al., 2011). The N-terminal-deleted PINK1 ( $\triangle \mathrm{N}$-PINK1) is degraded by the $\mathrm{N}$-end rule pathway and the ubiquitin proteasome system (Pickrell and Youle, 2015). However, under various stressors or MMP fluctuations, PINK1 is shunted and retained on the outer mitochondrial membrane (OMM), promoting Parkin recruitment to the defective mitochondrial surface with the help of PINK1 autophosphorylation (Hasson et al., 2013; Lazarou et al., 2015). Parkin, an E3 ubiquitin ligase, ubiquitinates several OMM proteins, including voltage-dependent anion-selective channel protein (VDAC), mitofusin 2 (Mfn2), and dynamin-1-like protein (DRP1), leading to their recognition by autophagic adaptors: OPTN, NDP52, sequestosome 1 (SQSTM1/p62), TAX1BP1, or NBR1 (Sarraf et al., 2013; Lazarou et al., 2015; Ordureau et al., 2018).

Growing evidence indicates the existence and importance of PINK1- and/or Parkin-independent pathways. In addition to Parkin, other E3 ubiquitin ligases, such as mitochondrial ubiquitin ligase activator of NF-kB1 (MUL1), seven in absentia homolog 1 (SIAH1), Gp78, SMAD ubiquitin regulatory factor 1 (SMURF1), and Ariadne RBR E3 ubiquitin protein ligase 1 (ARIH1) participate in mitophagy. These E3 ubiquitin ligases are localized on OMM to generate ubiquitin chains, in order to direct coupling to the autophagy protein LC3, enabling the engulfment of the ubiquitin chain-tagged mitochondria by phagosomes, and finally fusion with the acidic lysosome to degrade the damaged mitochondria (Szargel et al., 2016; Villa et al., 2017). In addition to ubiquitin ligase-dependent mitophagy, OMM proteins can act as mitophagy receptors, targeting damaged mitochondria directly for mitophagy-mediated degradation. Examples include: BNIP3, NIX/BNIP3L, and FUNDC1 that mediate mitochondrial elimination via display of the $\mathrm{N}$-terminus LIR domain into the cytosol which interact with LC3 or gammaaminobutyric acid receptor-associated protein (GABARAP) (Sandoval et al., 2008; Liu et al., 2012; Zhang et al., 2016; Palikaras et al., 2018; Villa et al., 2018; Lou et al., 2019). Additionally, PHB2 and cardiolipin are amongst the recently discovered mitophagy proteins, which can externalized to OMM and couple with LC3 following mitochondrial membrane depolarization (Shen et al., 2017; Wei et al., 2017). In summary, while the PINK1/Parkin-dependent mitophagy pathway is well-characterized, the molecular mechanisms of multiple new mitophagy pathways are still not fully understood (Figure 1).

\section{DEFECTIVE MITOPHAGY IN AD}

Whilst accumulated extracellular $\mathrm{A} \beta$ plaques and intraneuronal Tau tangles are the disease-defining pathological features of Alzheimer's disease (AD), inflammation is now widely recognized as a key additional hallmark of AD. Relationships between mitophagy and each of the hallmarks of $\mathrm{AD}$ are summarized below.

\section{Mitophagy and Amyloid- $\beta$ ( $A \beta)$}

Neurons affected in AD models undergo defective mitophagy that contribute to the disease-defining $A \beta$ pathologies, while $\mathrm{A} \beta$ accumulation may exacerbate impaired mitophagy and vice versa (Du et al., 2017; Kerr et al., 2017; Fang, 2019; Fang et al., 2019). Impaired mitochondrial proteostasis, including impaired mitochondrial unfolded protein response $\left(\mathrm{UPR}^{m t}\right)$, may link to $A \beta$ proteotoxicity (Sorrentino et al., 2017). The activating transcription factor-associated with stress (ATFS-1) protein plays a fundamental role in the maintenance of $\mathrm{UPR}^{m t}$ and mitochondrial function, especially in stress conditions (Nargund et al., 2012). RNAi knockdown of atfs-1 in an A $\beta$ Caenorhabditis elegans model (GMC101) repressed mitophagy as well as basal and maximal respiration, and exacerbated $\mathrm{A} \beta$ toxicity; However, restoration of $\mathrm{UPR}^{m t}$ diminished $\mathrm{AD}$ pathology in both $C$. elegans and mouse models of $\mathrm{AD}$ (Sorrentino et al., 2017). Mechanistically, ATFS-1 transfers into and is degraded within mitochondrial matrix, which negatively impacts UPR ${ }^{m t}$, at physiological condition (Melber and Haynes, 2018). Under the condition of mitochondrial stress, ATFS-1 favors importation into the nucleus, whereby it promotes the expression of genes with encoded proteins involved in the protection of mitochondrial function and the elimination of AD pathology (Melber and Haynes, 2018). In support of this model, mutations that cause amino acid substitutions within the MTS of ATFS-1 prevent the protein from being imported into the mitochondrial matrix, and result in constitutive $\mathrm{UPR}^{m t}$ activation (Rauthan et al., 2013). Abnormal mitochondrial homeostasis was reported in the mutant APP-HT22 cells relative to non-transfected HT22 cells, including increased levels of mitochondrial fission proteins (Drp1 and Fis1) and decreased levels of fusion proteins (Mfn1, Mfn2, and Opa1) (Manczak et al., 2018; Reddy et al., 2018). In addition to impaired $\mathrm{UPR}^{m t}$, defective mitophagy is another major cause of impaired mitochondrial proteostasis and $\mathrm{A} \beta$ proteinopathy in $\mathrm{AD}$. On one hand, defective mitophagy in post-mortem brain tissues from $\mathrm{AD}$ patients as well as in AD iPSC-derived neurons and crossspecies $\mathrm{A} \beta$-based $\mathrm{AD}$ animal models have been demonstrated (Fang et al., 2019). On the other, restoration of neuronal and microglial mitophagy ameliorated $\mathrm{A} \beta$ proteinopathy and rescued memory loss in the APP/PS1 mouse models of $\mathrm{AD}$, highlighting the important contribution of defective mitophagy in AD (Fang et al., 2019). Disrupted-in-schizophrenia-1 (DISC1), an LC3-binding mitophagy protein, has been shown 


Mitophagy
induction
(NR, NMN, UA, AC)

to be reduced in human AD brain samples and in the APP/PS1 mice. In fact, $A \beta$-induced mitochondrial dysfunction, loss of spines, and impaired long-term potentiation (LTP) were rescued upon DISC1 overexpression in the APP/PS1 mice (Wang et al., 2019). Collectively, the current studies implicate that impaired mitochondrial proteostasis as a contributor to $\mathrm{A} \beta$-based neurotoxicity via impaired $\mathrm{UPR}^{m t}$ and compromised mitophagy. However, the detailed molecular mechanisms remain to be determined.

\section{Mitophagy and Tau}

Tau binds and stabilizes microtubules, contributing in multiple physiological functions, such as neurite outgrowth, neuronal development, axonal transport, and synaptogenesis (Ballatore et al., 2007; Dixit et al., 2008). Studies in experimental AD models have provided evidence that mitochondrial dysfunction, defective mitophagy and phosphorylated-Tau ( $\mathrm{p}$-Tau) interact to form a vicious cycle (Kerr et al., 2017). The toxic $\mathrm{N}$-terminal truncation of human Tau $\left(\mathrm{NH}_{2}\right.$-hTau) strongly affects the interplay between the mitochondria dynamics and mitophagy affecting subcellular trafficking or recruitment of both Parkin and ubiquitin-C-terminal hydrolase L1 (UCHL1) (Amadoro et al., 2014; Corsetti et al., 2015). In C. elegans and neuroblastoma cells, expression of human wild-type (hTau) and frontotemporal dementia mutant tau (hP301L) completely inhibited mitophagy by blocking the recruitment of Parkin to damaged mitochondria (Cummins et al., 2019). Furthermore, APP and tau overexpression lead mitophagy impairment in human unmodified fibroblasts (Martin-Maestro et al., 2019). Furthermore, mitophagy was impaired in hippocampus tissues from 3xTgAD mice (with both $\mathrm{A} \beta$ and Tau proteinopathies) (Fang et al., 2019). In addition, pharmacological restoration of mitophagy, via administration of $\mathrm{NAD}^{+}$precursor nicotinamide mononucleotide $(\mathrm{NMN})$, urolithin A (UA), or actinonin (AC), reduced the phosphorylation of pTau at several sites (such as Thr181, Ser202/Thr205, Thr231, and Ser262) (Fang et al., 2019). Collectively, emerging evidence suggests that pathological Tau inhibits mitophagy, highlighting defective mitophagy as a novel therapeutic target for AD.

\section{Mitophagy and Inflammation}

Numerous preclinical and clinical studies have shown that immune activation in $\mathrm{AD}$, including microglia, and several cytokines, has the capacity to trigger and drive the pathophysiology of AD (Heppner et al., 2015). Mitochondrial stress leads to the release of damage-associated molecular patterns (DAMPs) which activate innate immunity, with the Cyclic GMP-AMP synthase (cGAS)-STING pathway as a central regulator of the type I interferon response to cytosolic DNA (Ishikawa and Barber, 2008; Ishikawa et al., 2009; Chen et al., 2016). Mitophagy mitigates inflammation through the restriction of inflammatory cytokine secretion and the regulation of immune cell homeostasis, correlating with the pathogenesis of autoimmune diseases at multiple levels (Xu et al., 2019). Multiple studies have demonstrated that PINK1 and Parkin regulate both innate and adaptive immunities. First of all, there is a strong inflammatory phenotype in both Pink $1^{-/-}$ and Parkin ${ }^{-/}$mice, both of which were central regulators in the mitophagy process. Furthermore, PINK1 and Parkin mitigated STING-induced inflammation and rescued the loss of dopaminergic neurons from the substantia nigra (SN) in both Pink $1^{-/-}$and Parkin ${ }^{-/-}$mice following exhaustive exercise (Sliter et al., 2018). Additionally, PINK1 and Parkin regulate immunity by repressing mitochondrial antigen presentation (MitAP) via mitochondria-derived vesicles (MDVs) (Matheoud et al., 2016). While the roles of STING and MitAP in the inflammation phenotype of $\mathrm{AD}$ is obscure, impairment of the PINK1/Parkin pathway in AD (Sliter et al., 2018; Fang et al., 2019), points to a possibility of an overlapping effect between PD and $\mathrm{AD}$. The concomitant and intertwined molecular pathways that link defective mitophagy to $\mathrm{A} \beta$ and Tau proteinopathies, and inflammation need further exploration. Lastly, restoration of neuronal mitophagy (through $\mathrm{NAD}^{+}$supplementation, UA, and $\mathrm{AC}$ ) reduced $\mathrm{AD}$ pathologies in the $\mathrm{APP} / \mathrm{PS} 1 \mathrm{AD}$ mice via 
enhanced microglial phagocytosis of extracellular $\mathrm{A} \beta$ plaques and the mitigation of pro-inflammatory cytokines released by continually activated microglia (Fang et al., 2019). Changes of mitophagy in $\mathrm{AD}$ astrocytes are elusive. It has been show that astrocytes play an important role in mitophagic degradation of damaged mitochondria from adjacent neurons (Davis et al., 2014), thus mitophagy induction may also improve different functions of astrocytes in AD. A recent development of a threedimensional (3D) human $\mathrm{AD}$ triculture model, with neurons, astrocytes, and microglia (Park et al., 2018), may enable the studies of cell type-specific mitophagy in an environment which mimic the human brain. Collectively, while defective mitophagy plays a pivotal role in $\mathrm{AD}$ progression, and turning up mitophagy forestalls $\mathrm{AD}$ pathology, further molecular mechanisms on how mitophagy induction impacts neurons, astrocytes and microglia are necessary.

\section{DEFECTIVE MITOPHAGY IN OTHER NEURODEGENERATIVE DISEASE}

PD is a progressive neurological disorder that observably impairs patients' ability to control body balance and movements due to lack of dopaminergic neurons in the substantia nigra (SN), which exhibits abnormal accumulation of $\alpha$-synuclein fibrils in their cell body and neurites (Poewe et al., 2017). Mitochondrial dysfunction and its related oxidative stress and inflammation are increasingly appreciated as common features of dopaminergic neuronal susceptibility in PD patient brain samples, PD animal models, and/or PD iPSC-derived neurons (Ryan et al., 2015; Schondorf et al., 2018). As a classical mitophagy pathway, the PINK1/Parkin pathway eliminates damaged mitochondria. Lossof-function mutations in PINK1 and/or PARK2/Parkin lead to inability of the cell to eliminate damaged mitochondria, and this has been related to early onset PD (Ryan et al., 2015). In addition, PINK1 and Parkin also suppress mitochondrial antigen presentation (MitAP) probably through inhibition of Sorting nexin 9 (Snx9)-dependent formation of MDVs (Matheoud et al., 2016). Meanwhile, Parkin- and Pink1-mutant fly models recapitulate major phenotypes of $\mathrm{PD}$, including mitochondrial dysfunction, dopaminergic neuronal loss, motor disabilities and reduced lifespan (Yang et al., 2006). For mice, while the Parkin $^{-/-}$and Pink1 $1^{-/-}$animals do not show PD phenotypes at standard laboratory living condition, they do exhibit PD phenotypes (e.g., the loss of dopaminergic neurons) at stress living conditions, such as intestinal infection, exhaustive exercise, and mitochondrial stress (Perez and Palmiter, 2005; McWilliams et al., 2018; Sliter et al., 2018; Matheoud et al., 2019). These rodent data suggest compensation of the loss of PINK1dependent mitophagy by PINK1-independent pathways under physiological conditions are sufficient; however, the PINK1pathway is necessary at stress/pathological conditions for the function and survival of PD-related dopaminergic neurons.

Amyotrophic lateral sclerosis (ALS) is a fatal neurodegenerative disease (predominately sporadic, nearly 90\%) characterized by the accumulation of aggregated proteins partially resulted from mitochondria dysfunction and oxidative stress within affected motor neurons in the spinal cord, brain stem, and motor cortex (Rowland and Shneider, 2001). Genetic studies of familial ALS have identified several genes linked to ALS (Cirulli et al., 2015). Most of the genes involved in cellular quality control pathways, and more specifically to selective autophagy and mitophagy, including mitophagy receptors OPTN, RIPK1, p62/SQSTM1, as well as TBK1 (Cirulli et al., 2015; Hawk et al., 2018). In this way, mutant OPTN and TBK1 can interfere with the process of mitophagy, while mutant p62 shows a lower affinity to LC3-II which leads to impaired mitophagy (Moore and Holzbaur, 2016). These data suggest that the inefficient turnover of damaged mitochondria and also aggregates, may contribute to disease progression in ALS (Weishaupt et al., 2016). In line with the argument that impaired autophagy/mitophagy as a driver of ALS, pharmacological or genetic up-regulation of the SIRT1/NAD ${ }^{+}$-mitophagy axis alleviates disease phenotypes in ALS mice and ALS patients (Blacher et al., 2019; de la Rubia et al., 2019; Lautrup et al., 2019). A detailed summary of defective mitophagy in AD, PD, ALS, and Huntington's disease is available (review in Lautrup et al., 2019; Lou et al., 2019). In summary, mounting evidence from animals and post-mortem human brain tissues suggests that defective mitophagy is a common feature, and likely plays a causative role in many neurodegenerative pathologies. We summarized the relationships between $\mathrm{AD}, \mathrm{PD}$, and ALS, and defective mitophagy/autophagy (Figure 2).

\section{MITOPHAGY INDUCERS}

Since reduced mitophagy is common in $\mathrm{AD}$, and maybe a causal mechanism, up-regulating mitophagy might provide a therapeutic strategy for AD (Kingwell, 2019). Small molecules that do not have toxicity to mitochondria (mitochondrial toxicants), but can induce the expression of mitophagy proteins or enhance mitophagy machinery hold translational promise (Ryu et al., 2016; Andreux et al., 2019; Fang, 2019; Lou et al., 2019). The classical mitochondrial uncouplers, e.g., carbonyl cyanide-p-(trifluoromethoxy)phenyl hydrazine (FCCP) and carbonyl cyanide m-chlorophenyl hydrazone (CCCP), and mitochondrial toxins that damage mitochondrial respiration (such as valinomycin, salinomycin, antimycin A and oligomycin) (Georgakopoulos et al., 2017), may have limited translational value for $\mathrm{AD}$ because treatment with those drugs will result in dysfunction of normal mitochondria.

In addition, multiple novel mitophagy inducers acting independently of the respiration failure without perturbing the organelle have been reported, offering new momentum to comprehend the process and underlying strategy for therapeutic revolution (Georgakopoulos et al., 2017). One example is to enhance the PINK1/Parkin-mediated mitophagy by supplementation with the ATP analog kinetin triphosphate (KTP) which can amplify catalytic activity of both PD related mutant PINK1 ${ }^{G 309 D}$ and PINK1 ${ }^{w t}$ (Hertz et al., 2013) or the application of a p53 inhibitor pifithrin-a, which can release Parkin from binding to the cytosolic p53 in pancreatic $\beta$-cells (Hoshino et al., 2014). Moreover, the anti-diabetic natural compound Metformin has been shown to maintain mitochondrial integrity 

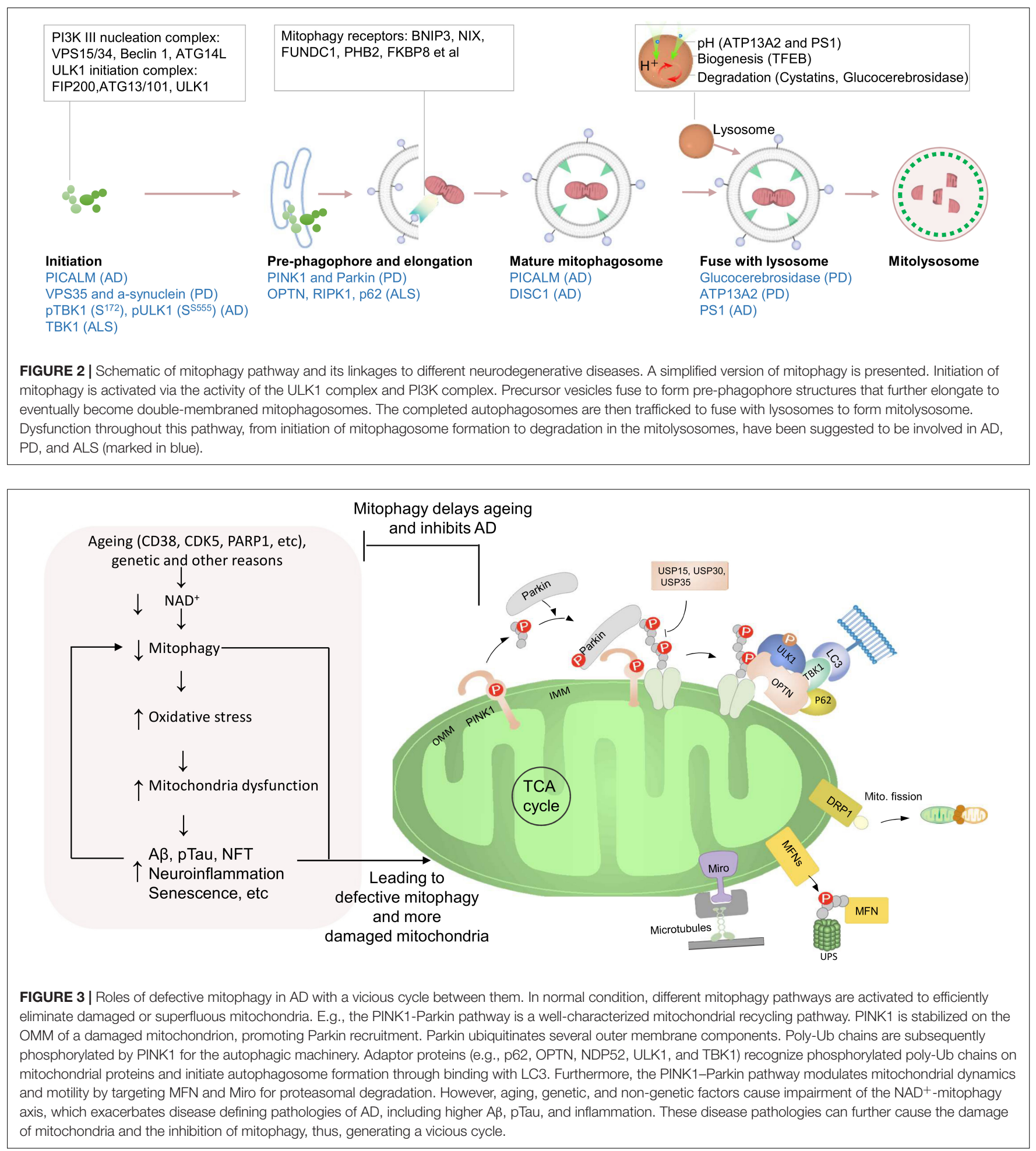

and boost mitochondrial biogenesis through Parkin-mediated mitophagy induction via p53 inhibition (Song et al., 2016; Palikaras et al., 2018). Targeting the up-regulation of the mammalian NF-E2 related factor 2 (Nrf2) (SKN-1, the C. elegans ortholog) pathway also enhances mitophagy, with molecules like the compound p62-mediated mitophagy inducer (PMI)
(East et al., 2014) and the natural compound Tomatidine affluent in the green tomato (Fang et al., 2017b). $\mathrm{NAD}^{+}$is a fundamental molecule in human health and life since it participates in glycolysis, TCA cycle, OXPHOS, $\beta$-oxidation, and many other bioenergetic and metabolic pathways (Verdin, 2015; Fang et al., 2017a; Aman et al., 2018; Mitchell et al., 2018). 
$\mathrm{NAD}^{+}$is reduced in biological aging, accelerated aging, and in common neurodegenerative diseases, including $\mathrm{AD}$ (Mouchiroud et al., 2013; Fang et al., 2014; Hou et al., 2018). Interventional studies support a causative role of $\mathrm{NAD}^{+}$ depletion in neurodegeneration, as augmentation of tissue $\mathrm{NAD}^{+}$, through the supplementation of nicotinamide riboside (NR) and NMN, can improve neuronal resilience and survival in both premature aging conditions and in $\mathrm{AD}$, through a mitophagy-dependent manner (Fang et al., 2014, 2016a, 2019). Mechanistically, $\mathrm{NAD}^{+}$induces mitophagy through the $\mathrm{NAD}^{+}$/Sirtuins-dependent pathways and several other pathways as we summarized elsewhere (Fang, 2019). In conclusion, small molecule which can induce mitophagy in vivo, but circumvent the cellular toxicity, hold promise for further clinical studies on AD.

\section{FUTURE PERSPECTIVES}

Accumulating data suggest the existence of PINK1/Parkindependent and -independent mitophagy pathways that are critical in the maintenance of mitochondrial homeostasis as well as neuronal resilience against proteinopathies and stressors. A growing understanding of AD pathology suggests that accumulation of damaged mitochondria due to impaired mitophagy, contributes to $\mathrm{A} \beta / \mathrm{Tau}$ proteinopathies and inflammation, which may ultimately lead to neuronal loss and memory impairment. Accordingly, experiments from C. elegans and mouse models of $\mathrm{AD}$ and from AD iPSCderived neurons suggest that turning up mitophagy might mitigate $\mathrm{AD}$ pathologies and retain cognition (in $\mathrm{AD}$ animals) with possible mechanisms summarized (Figure 3). Some outstanding questions need to be further addressed. First, whether defective mitophagy is an early event preceding and causing $A \beta /$ Tau proteinopathies? Second, what are the additional molecular mechanisms of defective mitophagy in AD? Cellular signaling and progresses, including histone modification and DNA methylation, DNA repair, senescence, and cell to cell communication (including neurons and glial cells) link to neural plasticity and cognitive function (Halder et al., 2016; Fang et al., 2019; Zhang et al., 2019). Possible linkages of mitophagy in these processes should be explored (Figure 1). Third, whether pharmacological restoration of mitophagy could rescue/delay the progression of memory loss in $\mathrm{AD}$ patients?

\section{REFERENCES}

Amadoro, G., Corsetti, V., Florenzano, F., Atlante, A., Ciotti, M. T., Mongiardi, M. P., et al. (2014). AD-linked, toxic NH2 human tau affects the quality control of mitochondria in neurons. Neurobiol. Dis. 62, 489-507. doi: 10.1016/j.nbd. 2013.10.018

Aman, Y., Frank, J., Lautrup, S. H., Matysek, A., Niu, Z., Yang, G., et al. (2019). The $\mathrm{NAD}(+)$-mitophagy axis in healthy longevity and in artificial intelligence-based clinical applications. Mech. Ageing Dev. 185:111194. doi: 10.1016/j.mad.2019. 111194

Aman, Y., Qiu, Y., Tao, J., and Fang, E. F. (2018). Therapeutic potential of boosting $\mathrm{NAD}+$ in aging and age-related diseases. Transl. Med. Aging 2, 30-37. doi: 10.1016/j.tma.2018.08.003
Because no single strategy has been effective in treating $\mathrm{AD}$, it is possible that a multi-targeted combinational approach, and even personalized treatments will be necessary to treating AD. Since long-term multidomain intervention, including intervention of diet, exercise and cognitive training, could improve or maintain cognitive functioning in at-risk elderly people from the general population, a role of mitophagy is worthy of further exploration (Ngandu et al., 2015). The availability of new experimental systems, including the AD patient-oriented neuronal and glial cells (Haenseler et al., 2017; Lin et al., 2018; Volpato et al., 2018), the 3D tri-culture system (Park et al., 2018), and the application of artificial intelligence (Aman et al., 2019) will enable mechanistic studies in models more closely resembling the human $\mathrm{AD}$ brain, and will propel drug development.

\section{AUTHOR CONTRIBUTIONS}

CX and EF designed the outline of the review and wrote the draft of the review. YA, BA, MC, HP-F, and JX provided scientific comments and wrote part of the review.

\section{FUNDING}

MC was supported by NIHR Oxford BRC. EF was supported by the HELSE SØR-ØST (\#2017056 and \#2020001), the Research Council of Norway (\#262175 and \#277813), the National Natural Science Foundation of China (\#81971327), an Akershus University Hospital Strategic grant (\#269901), and a Rosa Sløyfe grant (\#207819) by the Norwegian Cancer Society. CX was supported by National Natural Science Foundation of China (No. 81600977), Natural Science Foundation of Zhejiang Province (Y19H090059), and Wenzhou City Committee of Science and Technology (Y20180137).

\section{ACKNOWLEDGMENTS}

The authors acknowledge the valuable work of the many investigators whose published articles they were unable to cite owing to space limitations. The authors thank Drs. Sofie Lautrup and Guofeng Lou for reading of the manuscript.

Andreux, P. A., Blanco-Bose, W., Ryu, D., Burdet, F., Ibberson, M., Aebischer, P., et al. (2019). The mitophagy activator urolithin $A$ is safe and induces a molecular signature of improved mitochondrial and cellular health in humans. Nat. Metab. 1, 593-603.

Ballatore, C., Lee, V. M., and Trojanowski, J. Q. (2007). Tau-mediated neurodegeneration in Alzheimer's disease and related disorders. Nat. Rev. Neurosci. 8, 663-672. doi: 10.1038/nrn2194

Blacher, E., Bashiardes, S., Shapiro, H., Rothschild, D., Mor, U., Dori-Bachash, M., et al. (2019). Potential roles of gut microbiome and metabolites in modulating ALS in mice. Nature 572, 474-480. doi: 10.1038/s41586-0191443-5

Burchell, V. S., Nelson, D. E., Sanchez-Martinez, A., Delgado-Camprubi, M., Ivatt, R. M., Pogson, J. H., et al. (2013). The Parkinson's disease-linked proteins 
Fbxo7 and Parkin interact to mediate mitophagy. Nat. Neurosci. 16, 1257-1265. doi: $10.1038 / \mathrm{nn} .3489$

Chen, Q., Sun, L., and Chen, Z. J. (2016). Regulation and function of the cGASSTING pathway of cytosolic DNA sensing. Nat. Immunol. 17, 1142-1149. doi: 10.1038/ni.3558

Cirulli, E. T., Lasseigne, B. N., Petrovski, S., Sapp, P. C., Dion, P. A., Leblond, C. S., et al. (2015). Exome sequencing in amyotrophic lateral sclerosis identifies risk genes and pathways. Science 347, 1436-1441. doi: 10.1126/science.aaa3650

Corsetti, V., Florenzano, F., Atlante, A., Bobba, A., Ciotti, M. T., Natale, F., et al. (2015). NH2-truncated human tau induces deregulated mitophagy in neurons by aberrant recruitment of Parkin and UCHL-1: implications in Alzheimer's disease. Hum. Mol. Genet. 24, 3058-3081. doi: 10.1093/hmg/dd v059

Cummins, N., Tweedie, A., Zuryn, S., Bertran-Gonzalez, J., and Götz, J. (2019). Disease-associated tau impairs mitophagy by inhibiting Parkin translocation to mitochondria. EMBO J. 38:e99360. doi: 10.15252/embj.20189 9360

Davis, C. H., Kim, K. Y., Bushong, E. A., Mills, E. A., Boassa, D., Shih, T., et al. (2014). Transcellular degradation of axonal mitochondria. Proc. Natl. Acad. Sci. U.S.A. 111, 9633-9638. doi: 10.1073/pnas.140465 1111

de la Rubia, J. E., Drehmer, E., Platero, J. L., Benlloch, M., CaplliureLlopis, J., Villaron-Casales, C., et al. (2019). Efficacy and tolerability of EH301 for amyotrophic lateral sclerosis: a randomized, doubleblind, placebo-controlled human pilot study. Amyotroph. Lateral Scler. Frontotemporal Degener. 20, 115-122. doi: 10.1080/21678421.2018.153 6152

Deas, E., Plun-Favreau, H., Gandhi, S., Desmond, H., Kjaer, S., Loh, S. H., et al. (2011). PINK1 cleavage at position $\mathrm{A} 103$ by the mitochondrial protease PARL. Hum. Mol. Genet. 20, 867-879. doi: 10.1093/hmg/dd q526

Dixit, R., Ross, J. L., Goldman, Y. E., and Holzbaur, E. L. (2008). Differential regulation of dynein and kinesin motor proteins by tau. Science 319, 1086-1089. doi: $10.1126 /$ science. 1152993

Du, F., Yu, Q., Yan, S., Hu, G., Lue, L. F., Walker, D. G., et al. (2017). PINK1 signalling rescues amyloid pathology and mitochondrial dysfunction in Alzheimer's disease. Brain 140, 3233-3251. doi: 10.1093/brain/aw $\mathrm{x} 258$

East, D. A., Fagiani, F., Crosby, J., Georgakopoulos, N. D., Bertrand, H., Schaap, M., et al. (2014). PMI: a DeltaPsim independent pharmacological regulator of mitophagy. Chem. Biol. 21, 1585-1596. doi: 10.1016/j.chembiol.2014. 09.019

Fang, E. F. (2019). Mitophagy and NAD(+) inhibit Alzheimer disease. Autophagy 15, 1112-1114. doi: 10.1080/15548627.2019.1596497

Fang, E. F., Hou, Y., Palikaras, K., Adriaanse, B. A., Kerr, J. S., Yang, B., et al. (2019). Mitophagy inhibits amyloid-beta and tau pathology and reverses cognitive deficits in models of Alzheimer's disease. Nat. Neurosci. 22, 401-412. doi: 10. 1038/s41593-018-0332-9

Fang, E. F., Kassahun, H., Croteau, D. L., Scheibye-Knudsen, M., Marosi, K., Lu, H., et al. (2016a). $\mathrm{NAD}(+)$ replenishment improves lifespan and healthspan in ataxia telangiectasia models via mitophagy and DNA repair. Cell Metab. 24, 566-581. doi: 10.1016/j.cmet.2016.09.004

Fang, E. F., Scheibye-Knudsen, M., Chua, K. F., Mattson, M. P., Croteau, D. L., and Bohr, V. A. (2016b). Nuclear DNA damage signalling to mitochondria in ageing. Nat. Rev. Mol. Cell Biol. 17, 308-321. doi: 10.1038/nrm. 2016.14

Fang, E. F., Lautrup, S., Hou, Y. J., Demarest, T. G., Croteau, D. L., Mattson, M. P., et al. (2017a). NAD (+) in aging: molecular mechanisms and translational implications. Trends Mol. Med. 23, 899-916. doi: 10.1016/j.molmed.2017.08. 001

Fang, E. F., Waltz, T. B., Kassahun, H., Lu, Q., Kerr, J. S., Morevati, M., et al. (2017b). Tomatidine enhances lifespan and healthspan in C. elegans through mitophagy induction via the SKN-1/Nrf2 pathway. Sci. Rep. 7:46208. doi: 10. 1038/srep46208

Fang, E. F., Scheibye-Knudsen, M., Brace, L. E., Kassahun, H., SenGupta, T., Nilsen, H., et al. (2014). Defective mitophagy in XPA via PARP-1 hyperactivation and NAD(+)/SIRT1 reduction. Cell 157, 882-896. doi: 10.1016/j.cell.2014. 03.026
Fivenson, E. M., Lautrup, S., Sun, N., Scheibye-Knudsen, M., Stevnsner, T., Nilsen, H., et al. (2017). Mitophagy in neurodegeneration and aging. Neurochem. Int. 109, 202-209. doi: 10.1016/j.neuint.2017.02.007

Gandhi, S., Wood-Kaczmar, A., Yao, Z., Plun-Favreau, H., Deas, E., Klupsch, K., et al. (2009). PINK1-associated Parkinson's disease is caused by neuronal vulnerability to calcium-induced cell death. Mol. Cell. 33, 627-638. doi: 10.1016/ j.molcel.2009.02.013

Georgakopoulos, N. D., Wells, G., and Campanella, M. (2017). The pharmacological regulation of cellular mitophagy. Nat. Chem. Biol. 13, 136-146. doi: 10.1038/nchembio.2287

Gustafsson, A. B., and Dorn, G. W. (2019). Evolving and expanding the roles of mitophagy as a homeostatic and pathogenic process. Physiol. Rev. 99, 853-892. doi: 10.1152/physrev.00005.2018

Haenseler, W., Sansom, S. N., Buchrieser, J., Newey, S. E., Moore, C. S., Nicholls, F. J., et al. (2017). A highly efficient human pluripotent stem cell microglia model displays a neuronal-co-culture-specific expression profile and inflammatory response. Stem Cell Rep. 8, 1727-1742. doi: 10.1016/j.stemcr. 2017.05.017

Halder, R., Hennion, M., Vidal, R. O., Shomroni, O., Rahman, R. U., Rajput, A., et al. (2016). DNA methylation changes in plasticity genes accompany the formation and maintenance of memory. Nat. Neurosci. 19, 102-110. doi: 10.1038/nn.4194

Hasson, S. A., Kane, L. A., Yamano, K., Huang, C. H., Sliter, D. A., Buehler, E., et al. (2013). High-content genome-wide RNAi screens identify regulators of parkin upstream of mitophagy. Nature 504, 291-295. doi: 10.1038/nature1 2748

Hawk, M. A., Gorsuch, C. L., Fagan, P., Lee, C., Kim, S. E., Hamann, J. C., et al. (2018). RIPK1-mediated induction of mitophagy compromises the viability of extracellular-matrix-detached cells. Nat. Cell Biol. 20, 272-284. doi: 10.1038/ s41556-018-0034-2

Heppner, F. L., Ransohoff, R. M., and Becher, B. (2015). Immune attack: the role of inflammation in Alzheimer disease. Nat. Rev. Neurosci. 16, 358-372. doi: $10.1038 / \mathrm{nrn} 3880$

Hertz, N. T., Berthet, A., Sos, M. L., Thorn, K. S., Burlingame, A. L., Nakamura, K., et al. (2013). A neo-substrate that amplifies catalytic activity of parkinson'sdisease-related kinase PINK1. Cell 154, 737-747. doi: 10.1016/j.cell.2013.07. 030

Hoshino, A., Ariyoshi, M., Okawa, Y., Kaimoto, S., Uchihashi, M., Fukai, K., et al. (2014). Inhibition of $\mathrm{p} 53$ preserves Parkin-mediated mitophagy and pancreatic beta-cell function in diabetes. Proc. Natl. Acad. Sci. U.S.A. 111, 3116-3121. doi: 10.1073/pnas.1318951111

Hou, Y., Lautrup, S., Cordonnier, S., Wang, Y., Croteau, D. L., Zavala, E., et al. (2018). NAD $(+)$ supplementation normalizes key Alzheimer's features and DNA damage responses in a new AD mouse model with introduced DNA repair deficiency. Proc. Natl. Acad. Sci. U.S.A. 115, E1876-E1885. doi: 10.1073/pnas. 1718819115

Ishikawa, H., and Barber, G. N. (2008). STING is an endoplasmic reticulum adaptor that facilitates innate immune signalling. Nature 455, 674-678. doi: 10.1038/ nature 07317

Ishikawa, H., Ma, Z., and Barber, G. N. (2009). STING regulates intracellular DNAmediated, type I interferon-dependent innate immunity. Nature 461, 788-792. doi: 10.1038 /nature 08476

Kerr, J. S., Adriaanse, B. A., Greig, N. H., Mattson, M. P., Cader, M. Z., Bohr, V. A., et al. (2017). Mitophagy and Alzheimer's disease: cellular and molecular mechanisms. Trends Neurosci. 40, 151-166. doi: 10.1016/j.tins.2017. 01.002

Kingwell, K. (2019). Turning up mitophagy in Alzheimer disease. Nat. Rev. Drug Discov. doi: 10.1038/d41573-019-00035-6 [Epub ahead of print].

Lautrup, S., Sinclair, D. A., Mattson, M. P., and Fang, E. F. (2019). NAD(+) in brain aging and neurodegenerative disorders. Cell Metab. 30, 630-655. doi: 10.1016/j.cmet.2019.09.001

Lazarou, M., Sliter, D. A., Kane, L. A., Sarraf, S. A., Wang, C., Burman, J. L., et al. (2015). The ubiquitin kinase PINK1 recruits autophagy receptors to induce mitophagy. Nature 524, 309-314. doi: 10.1038/nature1 4893

Lin, Y. T., Seo, J., Gao, F., Feldman, H. M., Wen, H. L., Penney, J., et al. (2018). APOE4 causes widespread molecular and cellular alterations associated with Alzheimer's Disease phenotypes in human iPSC-Derived 
brain cell types. Neuron 98, 1141.e7-1154.e7. doi: 10.1016/j.neuron.2018. 05.008

Liu, L., Feng, D., Chen, G., Chen, M., Zheng, Q., Song, P., et al. (2012). Mitochondrial outer-membrane protein FUNDC1 mediates hypoxia-induced mitophagy in mammalian cells. Nat. Cell Biol. 14, 177-185. doi: 10.1038/ ncb2422

Lou, G., Palikaras, K., Lautrup, S., Scheibye-Knudsen, M., Tavernarakis, N., Fang, E. F., et al. (2019). Mitophagy and neuroprotection. Trends Mol. Med. 26, 8-20. doi: 10.1016/j.molmed.2019.07.002

Manczak, M., Kandimalla, R., Yin, X., and Reddy, P. H. (2018). Hippocampal mutant APP and amyloid beta-induced cognitive decline, dendritic spine loss, defective autophagy, mitophagy and mitochondrial abnormalities in a mouse model of Alzheimer's disease. Hum. Mol. Genet. 27, 1332-1342. doi: 10.1093/ hmg/ddy042

Martin-Maestro, P., Gargini, R., Garcia, E., Simon, D., Avila, J., and GarciaEscudero, V. (2019). Mitophagy failure in APP and Tau overexpression model of Alzheimer's disease. J. Alzheimers Dis. 70, 525-540. doi: 10.3233/JAD-190086

Matheoud, D., Cannon, T., Voisin, A., Penttinen, A. M., Ramet, L., Fahmy, A. M., et al. (2019). Intestinal infection triggers Parkinson's disease-like symptoms in Pink1(-/-) mice. Nature 571, 565-569. doi: 10.1038/s41586-019-1405-y

Matheoud, D., Sugiura, A., Bellemare-Pelletier, A., Laplante, A., Rondeau, C., Chemali, M., et al. (2016). Parkinson's disease-related proteins PINK1 and parkin repress mitochondrial antigen presentation. Cell 166, 314-327. doi: 10. 1016/j.cell.2016.05.039

McWilliams, T. G., Prescott, A. R., Allen, G. F., Tamjar, J., Munson, M. J., Thomson, C., et al. (2016). mito-QC illuminates mitophagy and mitochondrial architecture in vivo. J. Cell Biol. 214, 333-345. doi: 10.1083/jcb.20160 3039

McWilliams, T. G., Prescott, A. R., Montava-Garriga, L., Ball, G., Singh, F., Barini, E., et al. (2018). Basal mitophagy occurs independently of PINK1 in mouse tissues of high metabolic demand. Cell Metab. 27, 439.e5-449.e5. doi: 10.1016/ j.cmet.2017.12.008

Melber, A., and Haynes, C. M. (2018). UPR(mt) regulation and output: a stress response mediated by mitochondrial-nuclear communication. Cell Res. 28, 281-295. doi: 10.1038/cr.2018.16

Mitchell, S. J., Bernier, M., Aon, M. A., Cortassa, S., Kim, E. Y., Fang, E. F., et al. (2018). Nicotinamide improves aspects of healthspan, but not lifespan, in mice. Cell Metab. 27, 667.e4-676.e4. doi: 10.1016/j.cmet.2018.02.001

Moore, A. S., and Holzbaur, E. L. (2016). Dynamic recruitment and activation of ALS-associated TBK1 with its target optineurin are required for efficient mitophagy. Proc. Natl. Acad. Sci. U.S.A. 113, E3349-E3358. doi: 10.1073/pnas. 1523810113

Mouchiroud, L., Houtkooper, R. H., Moullan, N., Katsyuba, E., Ryu, D., Canto, C., et al. (2013). The NAD $(+) /$ Sirtuin pathway modulates longevity through activation of mitochondrial UPR and FOXO signaling. Cell 154, 430-441. doi: 10.1016/j.cell.2013.06.016

Nargund, A. M., Pellegrino, M. W., Fiorese, C. J., Baker, B. M., and Haynes, C. M. (2012). Mitochondrial import efficiency of ATFS-1 regulates mitochondrial UPR activation. Science 337, 587-590. doi: 10.1126/science. 1223560

Ngandu, T., Lehtisalo, J., Solomon, A., Levalahti, E., Ahtiluoto, S., Antikainen, R., et al. (2015). A 2 year multidomain intervention of diet, exercise, cognitive training, and vascular risk monitoring versus control to prevent cognitive decline in at-risk elderly people (FINGER): a randomised controlled trial. Lancet 385, 2255-2263. doi: 10.1016/S0140-6736(15)60461-5

Ordureau, A., Paulo, J. A., Zhang, W., Ahfeldt, T., Zhang, J., Cohn, E. F., et al. (2018). Dynamics of PARKIN-dependent mitochondrial ubiquitylation in induced neurons and model systems revealed by digital snapshot proteomics. Mol. Cell 70, 211.e8-227.e8. doi: 10.1016/j.molcel.2018.03.012

Palikaras, K., Lionaki, E., and Tavernarakis, N. (2018). Mechanisms of mitophagy in cellular homeostasis, physiology and pathology. Nat. Cell Biol. 20, 1013-1022. doi: 10.1038/s41556-018-0176-2

Park, J., Wetzel, I., Marriott, I., Dreau, D., D’Avanzo, C., Kim, D. Y., et al. (2018). A 3D human triculture system modeling neurodegeneration and neuroinflammation in Alzheimer's disease. Nat. Neurosci. 21, 941-951. doi: 10.1038/s41593-018-0175-4

Perez, F. A., and Palmiter, R. D. (2005). Parkin-deficient mice are not a robust model of parkinsonism. Proc. Natl. Acad. Sci. U.S.A. 102, 2174-2179. doi: 10.1073/pnas.0409598102
Pickrell, A. M., and Youle, R. J. (2015). The roles of PINK1, parkin, and mitochondrial fidelity in Parkinson's disease. Neuron 85, 257-273. doi: 10.1016/ j.neuron.2014.12.007

Plun-Favreau, H., and Hardy, J. (2008). PINK1 in mitochondrial function. Proc. Natl. Acad. Sci. U.S.A. 105, 11041-11042.

Poewe, W., Seppi, K., Tanner, C. M., Halliday, G. M., Brundin, P., Volkmann, J., et al. (2017). Parkinson disease. Nat. Rev. Dis. Primers 3:17013. doi: 10.1038/ nrdp. 2017.13

Princely Abudu, Y., Pankiv, S., Mathai, B. J., Hakon Lystad, A., Bindesboll, C., Brenne, H. B., et al. (2019). NIPSNAP1 and NIPSNAP2 act as "Eat Me" signals for mitophagy. Dev. Cell 49, 509.e12-525.e12. doi: 10.1016/j.devcel.2019.03.013

Rauthan, M., Ranji, P., Aguilera Pradenas, N., Pitot, C., and Pilon, M. (2013). The mitochondrial unfolded protein response activator ATFS-1 protects cells from inhibition of the mevalonate pathway. Proc. Natl. Acad. Sci. U.S.A. 110, 5981-5986. doi: 10.1073/pnas.1218778110

Reddy, P. H., Yin, X., Manczak, M., Kumar, S., Pradeepkiran, J. A., Vijayan, M., et al. (2018). Mutant APP and amyloid beta-induced defective autophagy, mitophagy, mitochondrial structural and functional changes and synaptic damage in hippocampal neurons from Alzheimer's disease. Hum. Mol. Genet. 27, 2502-2516. doi: 10.1093/hmg/ddy154

Rowland, L. P., and Shneider, N. A. (2001). Amyotrophic lateral sclerosis. N. Engl. J. Med. 344, 1688-1700.

Ryan, B. J., Hoek, S., Fon, E. A., and Wade-Martins, R. (2015). Mitochondrial dysfunction and mitophagy in Parkinson's: from familial to sporadic disease. Trends Biochem. Sci. 40, 200-210. doi: 10.1016/j.tibs.2015.02.003

Ryu, D., Mouchiroud, L., Andreux, P. A., Katsyuba, E., Moullan, N., Nicolet-DitFelix, A. A., et al. (2016). Urolithin A induces mitophagy and prolongs lifespan in C. elegans and increases muscle function in rodents. Nat. Med. 22, 879-888. doi: $10.1038 / \mathrm{nm} .4132$

Sandoval, H., Thiagarajan, P., Dasgupta, S. K., Schumacher, A., Prchal, J. T., Chen, M., et al. (2008). Essential role for Nix in autophagic maturation of erythroid cells. Nature 454, 232-235. doi: 10.1038/nature 07006

Sarraf, S. A., Raman, M., Guarani-Pereira, V., Sowa, M. E., Huttlin, E. L., Gygi, S. P., et al. (2013). Landscape of the PARKIN-dependent ubiquitylome in response to mitochondrial depolarization. Nature 496, 372-376. doi: 10.1038/nature12043

Scheibye-Knudsen, M., Fang, E. F., Croteau, D. L., Wilson, D. M., and Bohr, V. A. (2015). Protecting the mitochondrial powerhouse. Trends Cell Biol. 25, 158-170. doi: 10.1016/j.tcb.2014.11.002

Schondorf, D. C., Ivanyuk, D., Baden, P., Sanchez-Martinez, A., De Cicco, S., Yu, C., et al. (2018). The NAD+ precursor nicotinamide riboside rescues mitochondrial defects and neuronal loss in iPSC and fly models of Parkinson's disease. Cell Rep. 23, 2976-2988. doi: 10.1016/j.celrep.2018. 05.009

Shen, Z. N., Li, Y. R., Gasparski, A. N., Abeliovich, H., and Greenberg, M. L. (2017). Cardiolipin regulates mitophagy through the protein kinase C Pathway. J. Biol. Chem. 292, 2916-2923. doi: 10.1074/jbc.M116.753574

Sliter, D. A., Martinez, J., Hao, L., Chen, X., Sun, N., Fischer, T. D., et al. (2018). Parkin and PINK1 mitigate STING-induced inflammation. Nature 561, 258-262. doi: 10.1038/s41586-018-0448-9

Song, Y. M., Lee, W. K., Lee, Y. H., Kang, E. S., Cha, B. S., and Lee, B. W. (2016). Metformin restores parkin-mediated mitophagy, suppressed by cytosolic p53. Int. J. Mol. Sci. 17:E122. doi: 10.3390/ijms17010122

Sorrentino, V., Romani, M., Mouchiroud, L., Beck, J. S., Zhang, H., Moullan, N., et al. (2017). Enhancing mitochondrial proteostasis reduces amyloid- $\beta$ proteotoxicity. Nature 552, 187-193. doi: 10.1038/nature25143

Szargel, R., Shani, V., Abd Elghani, F., Mekies, L. N., Liani, E., Rott, R., et al. (2016). The PINK1, synphilin-1 and SIAH-1 complex constitutes a novel mitophagy pathway. Hum. Mol. Genet. 25, 3476-3490. doi: 10.1093/hmg/ddw189

Verdin, E. (2015). NAD (+) in aging, metabolism, and neurodegeneration. Science 350, 1208-1213. doi: 10.1126/science.aac4854

Villa, E., Marchetti, S., and Ricci, J. E. (2018). No parkin zone: mitophagy without parkin. Trends Cell Biol. 28, 882-895. doi: 10.1016/j.tcb.2018.07.004

Villa, E., Proics, E., Rubio-Patino, C., Obba, S., Zunino, B., Bossowski, J. P., et al. (2017). Parkin-independent mitophagy controls chemotherapeutic response in cancer cells. Cell Rep. 20, 2846-2859. doi: 10.1016/j.celrep.2017.08.087

Volpato, V., Smith, J., Sandor, C., Ried, J. S., Baud, A., Handel, A., et al. (2018). Reproducibility of molecular phenotypes after long-term differentiation to 
human iPSC-derived neurons: a multi-site omics study. Stem Cell Rep. 11, 897-911. doi: 10.1016/j.stemcr.2018.08.013

Wang, Z. T., Lu, M. H., Zhang, Y., Ji, W. L., Lei, L., Wang, W., et al. (2019). Disrupted-in-schizophrenia-1 protects synaptic plasticity in a transgenic mouse model of Alzheimer's disease as a mitophagy receptor. Aging Cell 18:e12860. doi: $10.1111 /$ acel.12860

Wei, Y. J., Chiang, W. C., Sumpter, R., Mishra, P., and Levine, B. (2017). Prohibitin 2 is an inner mitochondrial membrane mitophagy receptor. Cell 168, 224.e10238.e10. doi: $10.1016 /$ j.cell.2016.11.042

Weishaupt, J. H., Hyman, T., and Dikic, I. (2016). Common molecular pathways in amyotrophic lateral sclerosis and frontotemporal dementia. Trends Mol. Med. 22, 769-783. doi: 10.1016/j.molmed.2016.07.005

Xu, Y., Shen, J., and Ran, Z. (2019). Emerging views of mitophagy in immunity and autoimmune diseases. Autophagy doi: 10.1080/15548627.2019.1603547 [Epub ahead of print].

Yang, Y., Gehrke, S., Imai, Y., Huang, Z., Ouyang, Y., Wang, J. W., et al. (2006). Mitochondrial pathology and muscle and dopaminergic neuron degeneration caused by inactivation of Drosophila Pink1 is rescued by Parkin. Proc. Natl. Acad. Sci. U.S.A. 103, 10793-10798. doi: 10.1073/pnas.060249 3103

Zhang, P., Kishimoto, Y., Grammatikakis, I., Gottimukkala, K., Cutler, R. G., Zhang, S., et al. (2019). Senolytic therapy alleviates Abeta-associated oligodendrocyte progenitor cell senescence and cognitive deficits in an Alzheimer's disease model. Nat. Neurosci. 22, 719-728. doi: 10.1038/s41593019-0372-9

Zhang, T., Xue, L., Li, L., Tang, C., Wan, Z., Wang, R., et al. (2016). BNIP3 protein suppresses PINK1 kinase proteolytic cleavage to promote mitophagy. J. Biol. Chem. 291, 21616-21629. doi: 10.1074/jbc.m116.733410

Conflict of Interest: EF has CRADA arrangements with ChromaDex, and is a consultant to Aladdin Healthcare Technologies and the Vancouver Dementia Prevention Centre.

The remaining authors declare that the research was conducted in the absence of any commercial or financial relationships that could be construed as a potential conflict of interest.

Copyright () 2020 Xie, Aman, Adriaanse, Cader, Plun-Favreau, Xiao and Fang. This is an open-access article distributed under the terms of the Creative Commons Attribution License (CC BY). The use, distribution or reproduction in other forums is permitted, provided the original author(s) and the copyright owner(s) are credited and that the original publication in this journal is cited, in accordance with accepted academic practice. No use, distribution or reproduction is permitted which does not comply with these terms. 